\title{
RANKL-Expressing Ectopic Extramammary Paget's Disease on the Lower Abdomen
}

\author{
Akiko Hagiwara-Takita Taku Fujimura Aya Kakizaki Setsuya Aiba \\ Department of Dermatology, Tohoku University Graduate School of Medicine, \\ Sendai, Japan
}

\section{Keywords}

Extramammary Paget's disease $\cdot$ RANKL $\cdot$ MMP7 $\cdot$ MMP25 $\cdot$ CCL5

\begin{abstract}
Ectopic extramammary Paget's disease (EMPD) is a rare variant of EMPD that develops in nonapocrine regions. Since reports about ectopic EMPD are limited, little is known about the biological and immunological background of ectopic EMPD. In this report, we present a case of ectopic EMPD on the lower abdomen that expressed RANKL but lacked the expression of MMP7. As we previously reported, Paget's cells express RANKL and MMP7, release soluble RANKL in the tumor microenvironment, and stimulate tumor-associated macrophages to produce tumor-loading factors in conventional EMPD. In our present case, both CCL5expressing cells and MMP25-bearing cells were lacking, whereas substantial numbers of CCL5-expressing cells and MMP25-bearing cells were found in conventional EMPD. Our case suggested that the lack of MMP7 on Paget's cells might be one of the possible explanations for the biology of ectopic EMPD.

(C) 2016 The Author(s)

Published by S. Karger AG, Basel
\end{abstract}

\section{Introduction}

Ectopic extramammary Paget's disease (EMPD) is a rare variant of EMPD that develops in nonapocrine regions [1]. Since reports about ectopic EMPD are limited and, to our 
knowledge, only 15 cases have previously been reported in the English literature [1-4], little is known about the biological and immunological background of ectopic EMPD. In this report, we present a case of ectopic EMPD on the lower abdomen that expressed RANKL but lacked the expression of MMP7.

\section{Case Report}

A 66-year-old woman presented to our Department with a 8-month history of asymptomatic erythema on the lower abdomen. On her initial visit, physical examination revealed a brown-red plaque on the right side of the mons puvis (fig. 1A). The size of the tumor was approximately $20 \times 20 \mathrm{~mm}$ in diameter. Skin biopsy revealed rounded cells that were devoid of intracellular bridges and a large nucleus in the epidermis (fig. 1B). Immunohistochemical staining revealed that these tumor cells were positive for CK7, PAS, GCDFP-15, Alcian blue, as well as RANKL (fig. 2A), and negative for CK20 and MMP7 (fig. 2B). In addition, CD163+ tumor-associated macrophages (TAMs) were detected adjacent to the Paget's cells in the dermis (fig. 2C). From the above findings, we diagnosed this case as RANKL-expressing ectopic EMPD. There was no significant enlargement of the bilateral inguinal lymph nodes. We resected the tumor with a 1-cm surgical margin. We screened for a possible internal malignancy with computed tomography scan and found no evidence of metastasis.

To further investigate the immunological background of our case, we employed immunohistochemical staining of CCL17, CCL5, and MMP25, both of which are reported to be increased on macrophages by the stimulation of soluble (s)RANKL [5], for our present case and 5 cases of conventional EMPD. Substantial numbers of CCL17-expressing cells (fig. 3A), CCL5-expressing cells (fig. 3C), and MMP25-bearing cells (fig. 3E) were detected in the conventional EMPD, whereas few CCL17-expressing cells (fig. 3B), CCL5-expressing cells (fig. 3D), and MMP25-bearing cells (fig. 3F) were detected in the present case.

The following antibodies (Abs) were used for immunofluorescence staining: mouse monoclonal anti-human CD163 Ab (clone: 10D6; Novocastra, UK), and anti-human CCL5 Ab (clone: 50013-5; LifeSpan BioScience, Seattle, Wash., USA), rabbit polyclonal anti-human RANKL Ab (LifeSpan BioScience), anti-human MMP7 Ab (LifeSpan BioScience), and antihuman MMP25 Ab (Abcam, Tokyo, Japan), and goat polyclonal anti-human CCL17 Ab (R\&D Systems, Tokyo, Japan).

\section{Discussion}

Together with regulatory $\mathrm{T}$ cells (Tregs), TAMs play critical roles in maintaining the immunosuppressive tumor microenvironment by producing various chemokines, including Th2-related chemokines, such as CCL5 and CCL17, in the lesional skin of EMPD [5-7]. CCL5 is a chemokine that plays a role in polarizing naive T cells to Th2 cells through CCR3 signaling pathways [8]. CCL5/CCR3 signaling promotes metastasis by inducing the Th2 polarization of $\mathrm{CD} 4^{+} \mathrm{T}$ cells and determines the prognosis of luminal breast cancer [9]. On the other hand, CCL17 derived from TAMs stimulated by RANKL plays a crucial role in the recruitment of Tregs to the tumor site to maintain the immunosuppressive tumor microenvironment [8]. Taken together, TAMs in EMPD develop an immunosuppressive microenvironment by producing of tumor-loading chemokines. 
Among the series of matrix metalloproteinases (MMP), MMP25 is upregulated by the stimulation of RANKL on monocytes-derived macrophages [5]. MMP25 degrades types I-IV collagens, gelastin, fibronectin, and fibrin, and is reported to correlate with tumor migration, tumor invasion, and prognosis of several types of cancer $[10,11]$.

Unlike conventional EMPD, immunohistochemical staining in the present case revealed the expression of RANKL but the lack of expression of MMP7 on Paget's cells. As we previously reported, Paget's cells express RANKL and MMP7 [6, 12], release sRANKL in the tumor microenvironment [6], and stimulate TAMs to produce Tregs-related chemokines, such as CCL17, in conventional EMPD [7]. Therefore, the lack of MMP7 might abrogate the release of sRANKL, leading to the downregulation of chemokines and MMPs from TAMs. Indeed, CCL17 expressing cells, CCL5-expressing cells, and MMP25-bearing cells were lacking in the present case. Notably, to our knowledge, there is no report in the English literature that describes metastatic ectopic EMPD [1-4]. The lack of MMP7 on Paget's cells might be one of the possible explanations for the biology of ectopic EMPD. Since this report presents a single case, further analysis of the mechanisms underlying this phenomenon may provide fundamental insights into the biology of ectopic EMPD. Such issues will need to be clarified in future investigations.

\section{Statement of Ethics}

The patient gave written informed consent.

\section{Disclosure Statement}

The authors have no conflicts of interest to declare.

\section{References}

1 Sawada Y, Bito T, Kabashima R, Yoshiki R, Hino R, Nakamura M, Shiraishi M, Tokura Y: Ectopic extramammary Paget's disease: case report and literature review. Acta Derm Venereol 2010;90:502505.

2 Vincent J, Taube JM: Pigmented extramammary Paget disease of the abdomen: a potential mimicker of melanoma. Dermatol Online J 2011;17:13.

-3 Ximena E, del Puerto C, Mario P, Sergio G: Ectopic extramammary Paget's disease on the face. Indian J Dermatol Venereol Leprol 2012;78:760-762.

4 Kuniyuki S, Maekawa N: Ectopic extramammary Paget's disease on the head: case report and literature review. Int J Dermatol 2015;54:e483-e486.

-5 Fujimura T, Kambayashi Y, Furudate S, Kakizaki A, Hidaka T, Asano M, Aiba S: Receptor activator of nuclear factor kappa-B ligand (RANKL)/RANK signaling promotes cancer-related inflammation through M2 macrophages. Exp Dermatol 2016;25:397-399.

-6 KambayashiY, Fujimura T, Furudate S, et al: The possible interaction between receptor activator of nuclear factor kappa-B ligand (RANKL) expressed by extramammary Paget cells and its ligand on dermal macrophages. J Invest Dermatol 2015;35:2547-2550.

7 Fujimura T, Kambayashi Y, Furudate S, et al: Receptor activator of nuclear factor kappa-B ligand (RANKL) promotes the production of CCL17 from RANK+ M2 macrophages. J Invest Dermatol 2015;135:2884-2887.

8 Komohara Y, Fujiwara Y, Ohnishi K, Takeya M: Tumor-associated macrophages: potential therapeutic targets for anti-cancer therapy. Adv Drug Deliv Rev 2016;99:180-185.

-9 Zhang Q, Qin J, Zhong L, Gong L, Zhang B, Zhang Y, Gao WQ: CCL5-mediated Th2 immune polarization promotes metastasis in luminal breast cancer. Cancer Res 2015;75:4312-4321. 


\section{Case Reports in \\ Dermatology}

\begin{tabular}{l|l}
\hline Case Rep Dermatol 2016;8:130-135 \\
\hline $10.1159 / 000445992$ & $\begin{array}{l}\text { @ 2016 The Author(s). Published by S. Karger AG, Basel } \\
\text { www.karger.com/cde }\end{array}$ \\
\hline
\end{tabular}

Hagiwara-Takita et al.: RANKL-Expressing Ectopic Extramammary Paget's Disease on the Lower Abdomen

10 Wang Y, Yu SJ, Li YX, Luo HS: Expression and clinical significance of matrix metalloproteinase-17 and -25 in gastric cancer. Oncol Lett 2015;9:671-676.

-11 Mäkinen LK, Häyry V, Hagström J, Sorsa T, Passador-Santos F, Keski-Säntti H, Haukka J, Mäkitie AA, Haglund C, Atula T: Matrix metalloproteinase-7 and matrix metalloproteinase-25 in oral tongue squamous cell carcinoma. Head Neck 2014;36:1783-1788.

-12 Fujimura T, Furudate S, Kambayashi Y, Kakizaki A, Haga T, Hashimoto A, Aiba S: Multiple metastasized extramammary Paget's disease cured with bisphosphonate risedronate sodium after CyberKnife radiosurgery and docetaxel chemotherapy. Dermatol Sinica 2016, in press. DOI: http://dx.doi.org/10.1016/j.dsi.2016.02.001.
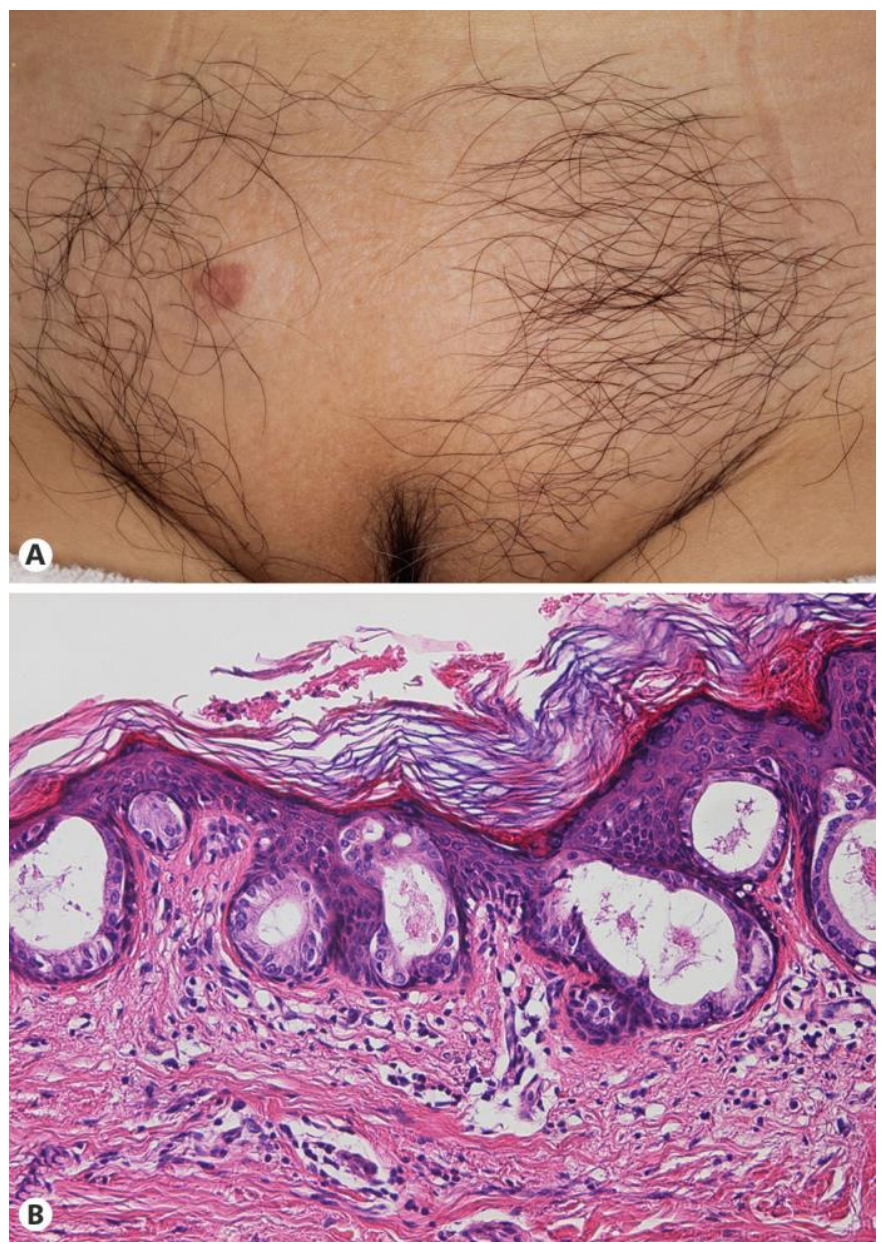

Fig. 1. A brown-red plaque on the right side of the mons puvis (A). Rounded cells that were devoid of intracellular bridges and large nucleus in the epidermis (B). Original magnification. $\times 200$ (B). 


\section{Case Reports in \\ Dermatology}

Case Rep Dermatol 2016;8:130-135

(C) 2016 The Author(s). Published by S. Karger AG, Basel

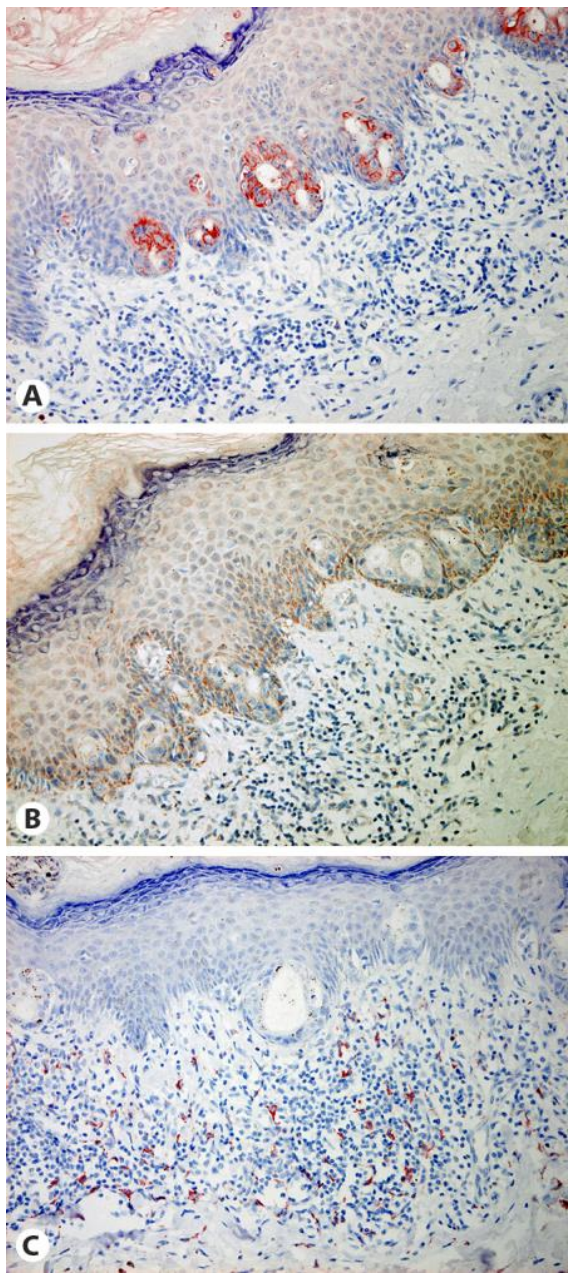

Fig. 2. Paraffin-embedded tissue samples were deparaffinized and stained with anti-RANKL Ab (A), antiMMP7 Ab (B), and anti-CD163 Ab (C). The sections were developed with liquid permanent red. Original magnification. $\times 200(A, B), \times 100(C)$.

Hagiwara-Takita et al.: RANKL-Expressing Ectopic Extramammary Paget's Disease on the Lower Abdomen 


\section{Case Reports in \\ Dermatology}

Case Rep Dermatol 2016;8:130-135

(C) 2016 The Author(s). Published by S. Karger AG, Basel
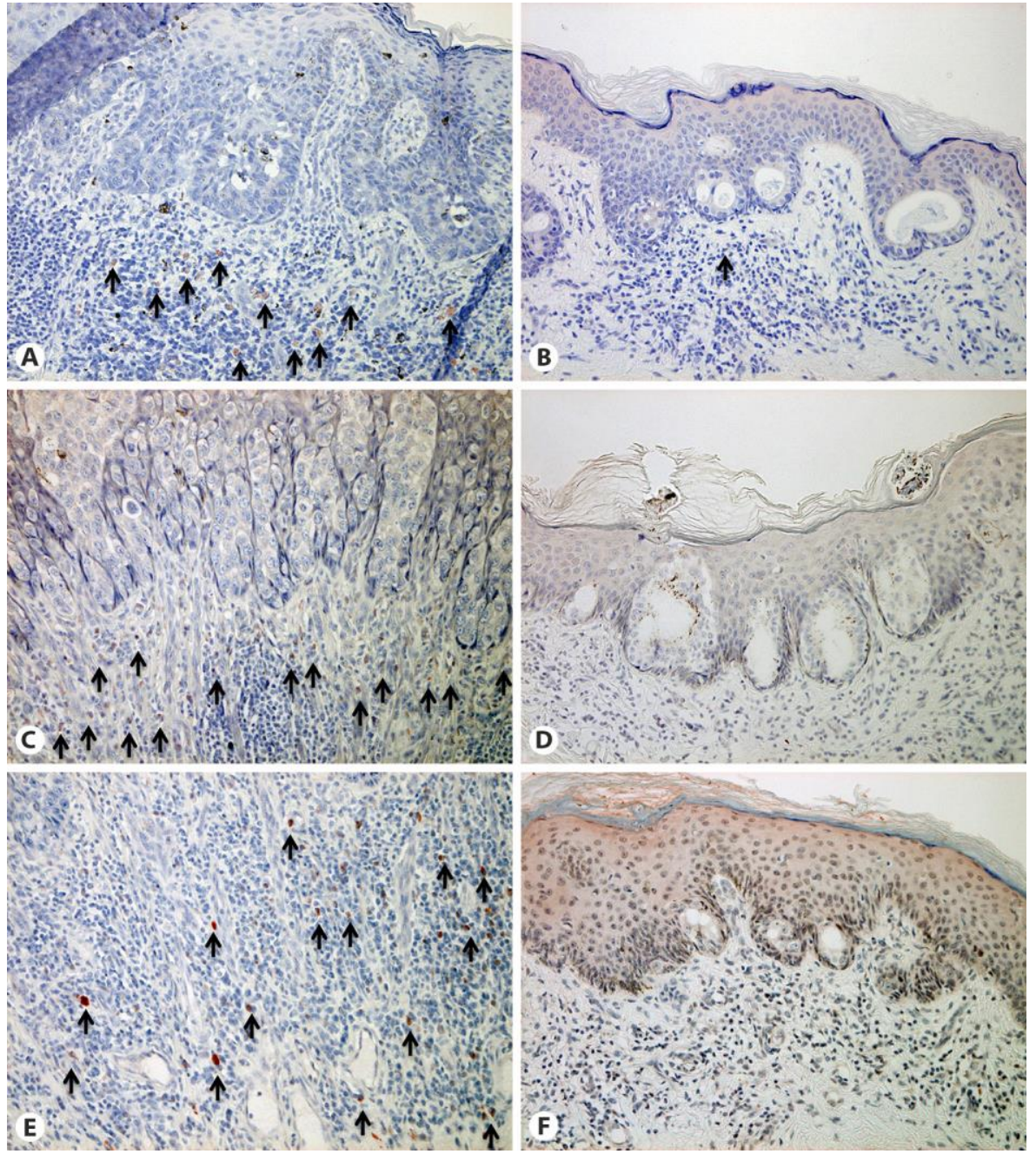

Fig. 3. Immunohistochemical staining for conventional EMPD (A, C, E) and ectopic EMPD (B, D, F). Paraffinembedded tissue samples were deparaffinized and stained with anti-CCL17 Ab (A, B), anti-CCL5 Ab (C, D), and anti-MMP25 Ab (E, F). The sections were developed with liquid permanent red. Original magnification. $\times 200$ (A, B, D), $\times 100$ (C). 